PROCEEDINGS OF THE

AMERICAN MATHEMATICAL SOCIETY

Volume 138, Number 7, July 2010, Pages 2301-2309

S 0002-9939(10)10303-7

Article electronically published on March 3, 2010

\title{
A BARBAN-DAVENPORT-HALBERSTAM ASYMPTOTIC FOR NUMBER FIELDS
}

\author{
ETHAN SMITH
}

(Communicated by Ken Ono)

\begin{abstract}
Let $K$ be a fixed number field, and assume that $K$ is Galois over $\mathbb{Q}$. Previously, the author showed that when estimating the number of prime ideals with norm congruent to $a$ modulo $q$ via the Chebotarëv Density Theorem, the mean square error in the approximation is small when averaging over all $q \leq Q$ and all appropriate $a$. In this article, we replace the upper bound by an asymptotic formula. The result is related to the classical Barban-DavenportHalberstam Theorem in the case $K=\mathbb{Q}$.
\end{abstract}

\section{INTRODUCTION}

One of the great results of the 1960s concerning the distribution of primes is that "on average" they are well-distributed in arithmetic progressions. In particular, Barban 1 and, independently, Davenport and Halberstam 2, 3 showed that the square of the error in the Prime Number Theorem for primes in arithmetic progressions is small on average. More precisely, given positive integers $a$ and $q$, we define the weighted prime counting function $\theta(x ; q, a)$ by

$$
\theta(x ; q, a):=\sum_{\substack{p \leq x \\ p \equiv a(\bmod q)}} \log p .
$$

The Prime Number Theorem for primes in arithmetic progressions states that if $\operatorname{gcd}(a, q)=1$, then

$$
\theta(x ; q, a) \sim \frac{x}{\varphi(q)},
$$

where $\varphi(q):=\#\{1 \leq a \leq q: \operatorname{gcd}(a, q)=1\}$ is Euler's $\varphi$-function. The BarbanDavenport-Halberstam Theorem (see [4]) states that, for any fixed $M>0$,

$$
\sum_{q \leq Q} \sum_{\substack{a=1 \\ \operatorname{gcd}(a, q)=1}}^{q}\left(\theta(x ; q, a)-\frac{x}{\varphi(q)}\right)^{2} \ll x Q \log x,
$$

provided that $x(\log x)^{-M} \leq Q \leq x$. Later, Montgomery [10] and Hooley [7] each gave asymptotic formulations of this result, valid for various ranges of $Q$. Hooley's

Received by the editors July 28, 2009, and, in revised form, October 15, 2009, and October 30, 2009.

2010 Mathematics Subject Classification. Primary 11N36, 11R44.

Key words and phrases. Generalized Siegel-Walfisz theorem, Barban-Davenport-Halberstam Theorem.

(c) 2010 American Mathematical Society Reverts to public domain 28 years from publication 
method starts with inequality (2), and so at least implicitly relies on the large sieve. Montgomery's method, however, is based on a result of Lavrik [9] concerning the distribution of twin primes.

With applications in mind, there have been several generalizations of this result to the integers of a number field. See [6, 13] for example. In [12, the author considered yet another generalization of (2) concerning the distribution of prime ideals of a number field. See Theorem 1 below. In the present article, we are concerned with the appropriate asymptotic formulation. See Theorem 2 .

\section{Statement of The MAIN TheOREM}

Let $K$ be a fixed number field. We are concerned with the error in estimating sums of the form

$$
\theta_{K}(x ; q, a):=\sum_{\substack{\mathrm{N} \mathfrak{p} \leq x, \mathrm{~Np} \equiv a(\bmod q)}} \log \mathrm{Np}
$$

via the Chebotarëv Density Theorem. Here, as usual, $\mathfrak{p}$ denotes a prime ideal of the ring of integers $\mathcal{O}_{K}$, and $\mathrm{Np}:=\#\left(\mathcal{O}_{K} / \mathfrak{p}\right)$ denotes its norm.

Let $\zeta_{q}$ be a primitive $q$-th root of unity, and let $G_{q}$ denote the image of the natural map

$$
\operatorname{Gal}\left(K\left(\zeta_{q}\right) / K\right) \hookrightarrow \operatorname{Gal}\left(\mathbb{Q}\left(\zeta_{q}\right) / \mathbb{Q}\right) \stackrel{\sim}{\longrightarrow}(\mathbb{Z} / q \mathbb{Z})^{*}
$$

In this case, the Frobenius substitution is determined by the value Np modulo $q$; and the Chebotarëv Density Theorem implies that if $a \in G_{q}$, then

$$
\theta_{K}(x ; q, a) \sim \frac{x}{\varphi_{K}(q)}
$$

where we have made the definition $\varphi_{K}(q):=\# G_{q}=\# \operatorname{Gal}\left(K\left(\zeta_{q}\right) / K\right)$.

If we assume further that $K / \mathbb{Q}$ is a Galois extension, then we have the following corollary of Goldstein's generalization of the Siegel-Walfisz Theorem [5]. If $a \in G_{q}$, then for any fixed $M>0$,

$$
\theta_{K}(x ; q, a)=\frac{x}{\varphi_{K}(q)}+O\left(\frac{x}{(\log x)^{M}}\right),
$$

provided that $q \leq(\log x)^{M}$. The following average error result is the main theorem of [12, where we continue to assume that our number field $K$ is a Galois extension of $\mathbb{Q}$.

Theorem 1. For a fixed $M>0$,

$$
\sum_{q \leq Q} \sum_{a \in G_{q}}\left(\theta_{K}(x ; q, a)-\frac{x}{\varphi_{K}(q)}\right)^{2} \ll x Q \log x
$$

if $x(\log x)^{-M} \leq Q \leq x$.

Remark. To be precise, the main theorem of [12] is stated in terms of

$$
\psi_{K}(x ; q, a):=\sum_{\substack{\mathrm{Np}^{m} \leq x, \mathrm{~Np}^{m} \equiv a(\bmod q)}} \log \mathrm{Np} .
$$

As usual, the statement and proof of the theorem is virtually unchanged when replacing $\psi_{K}(x ; q, a)$ by $\theta_{K}(x ; q, a)$. 
In this article, we continue to assume that $K / \mathbb{Q}$ is Galois and replace the inequality in Theorem 1 by an asymptotic formula. In particular, we show the following.

Theorem 2. For a fixed $M>0$,

(5) $\sum_{q \leq x} \sum_{a \in G_{q}}\left(\theta_{K}(x ; q, a)-\frac{x}{\varphi_{K}(q)}\right)^{2}=[K: \mathbb{Q}] x^{2} \log x+C_{1} x^{2}+O\left(\frac{x^{2}}{(\log x)^{M}}\right)$;

and if $1 \leq Q \leq x$,

(6)

$$
\begin{aligned}
\sum_{q \leq Q} \sum_{a \in G_{q}}\left(\theta_{K}(x ; q, a)-\frac{x}{\varphi_{K}(q)}\right)^{2}=[K: & \mathbb{Q}] x Q \log x-\frac{\varphi\left(m_{K}\right)}{\varphi_{K}\left(m_{K}\right)} x Q \log (x / Q) \\
& +C_{2} Q x+O\left(x^{3 / 4} Q^{5 / 4}+\frac{x^{2}}{(\log x)^{M}}\right),
\end{aligned}
$$

where $\varphi$ denotes the ordinary Euler $\varphi$-function, $C_{1}, C_{2}$ are constants, and $m_{K}$ is an integer defined in the first paragraph of Section 3 .

Remark. The constants $C_{1}, C_{2}$ appearing in the statement of the theorem depend on $K$ and may be given explicitly. However, the expressions are somewhat messy. For example, $C_{1}$ is given by

$$
C_{1}=F(1) \zeta^{\prime}(2)+F(1) \frac{(2 \gamma-3) \pi^{2}}{12}+F(1) F^{\prime}(1) \frac{\pi^{2}}{6}-[K: \mathbb{Q}] .
$$

Here, $\zeta(s)$ denotes the Riemann zeta function, $\gamma \approx 0.577$ is the Euler-Mascheroni constant, and $F(s):=h(s) \prod_{\ell \mid m_{K}} D_{K, \ell}(s)$. The functions $h(s)$ and $D_{K, \ell}(s)$ are described in Section 3

Remark. In the case that $K / \mathbb{Q}$ is Abelian, it turns out that $\varphi\left(m_{K}\right) / \varphi_{K}\left(m_{K}\right)=$ $[K: \mathbb{Q}]$. See the first paragraph of Section 3 Thus, in this case, equation (6) simplifies nicely to

$$
\begin{aligned}
\sum_{q \leq Q} \sum_{a \in G_{q}}\left(\theta_{K}(x ; q, a)-\frac{x}{\varphi_{K}(q)}\right)^{2}= & {[K: \mathbb{Q}] x Q \log Q+C_{2} Q x } \\
& +O\left(x^{3 / 4} Q^{5 / 4}+\frac{x^{2}}{(\log x)^{M}}\right) .
\end{aligned}
$$

Our proof of Theorem 2is an adaptation of Hooley's methods for the case $K=\mathbb{Q}$ as found in [7, pp. 209-212]. The proof will be carried out in Section 4.

\section{Preliminaries}

Before proceeding with the proof of Theorem 2 we first analyze the arithmetic function $\varphi_{K}(q)$. Let $\mathbb{Q}^{\text {cyc }}:=\bigcup_{q>1} \mathbb{Q}\left(\zeta_{q}\right)$, and let $\mathcal{A}:=\mathbb{Q}^{\text {cyc }} \cap K$. Then $\mathcal{A}$ is an Abelian extension of $\mathbb{Q}$ of finite degree. In particular, $\mathcal{A}$ is the maximal Abelian subfield of $K$. By the Kronecker-Weber Theorem, there exists a smallest integer $m_{K}$ such that $\mathcal{A} \subseteq \mathbb{Q}\left(\zeta_{m_{K}}\right)$ See, for example, [8, p. 210]. For each integer $q>0$, we define the intersection $A_{q}:=K \cap \mathbb{Q}\left(\zeta_{q}\right)$, whence, via restriction maps, $\operatorname{Gal}\left(K\left(\zeta_{q}\right) / K\right) \cong \operatorname{Gal}\left(\mathbb{Q}\left(\zeta_{q}\right) / A_{q}\right)$. Thus, it is clear that if $q$ is coprime to $m_{K}$, then $\varphi_{K}(q)=\varphi(q)$. In any case, $\varphi_{K}(q)$ is multiplicative and divides $\varphi(q)$. For each prime divisor $\ell$ of $m_{K}$, we define $b_{\ell}:=\operatorname{ord}_{\ell}\left(m_{K}\right)$, the order of $\ell$ dividing $m_{K}$. 
Lemma 1. For a prime $\ell, \varphi_{K}(\ell)$ is a divisor of $\ell-1$. In general, we have

$$
\varphi_{K}(q)=\prod_{\substack{\ell^{\alpha} \| q \\ \ell \nmid m_{K}}} \ell^{\alpha-1}(\ell-1) \prod_{\substack{\ell^{\alpha} \| q \\ \ell \mid m_{K} \\ \alpha \geq b_{\ell}}} \ell^{\alpha-b_{\ell}} \varphi_{K}(\ell) \prod_{\substack{\ell^{\alpha} \| q \\ \ell \mid m_{K} \\ \alpha<b_{\ell}}} \varphi_{K}(\ell) .
$$

Proof. The first statement is trivial, as $G_{q}$ is a subgroup of $(\mathbb{Z} / q \mathbb{Z})^{*}$. Since $\varphi_{K}(q)$ is multiplicative and $\varphi_{K}(q)=\varphi(q)$ for $\operatorname{gcd}\left(q, m_{K}\right)=1$, we restrict our attention to primes dividing $m_{K}$.

Suppose that $\ell$ is a prime dividing $m_{K}$. Then $A_{\ell^{b_{\ell}+k}}=A_{\ell^{b} \ell}$ for all integers $k \geq 0$. Thus, we immediately see that

$$
\varphi_{K}\left(\ell^{b_{\ell}+k}\right)=\left|\operatorname{Gal}\left(\mathbb{Q}\left(\zeta_{\ell^{b_{\ell}+k}}\right) / \mathbb{Q}\left(\zeta_{\ell^{b_{\ell}}}\right)\right)\right| \cdot\left|\operatorname{Gal}\left(\mathbb{Q}\left(\zeta_{\ell^{b_{\ell}}}\right) / A_{\ell^{b_{\ell}}}\right)\right|=\ell^{k} \varphi_{K}\left(\ell^{b_{\ell}}\right) .
$$

We claim that

$$
\varphi_{K}\left(\ell^{j}\right)=\varphi_{K}(\ell) \text { for } 1 \leq j \leq b_{\ell} .
$$

If $b_{\ell}=1$, the statement is trivial. Assume then that $b_{\ell} \geq 2$, and consider the following field diagram:

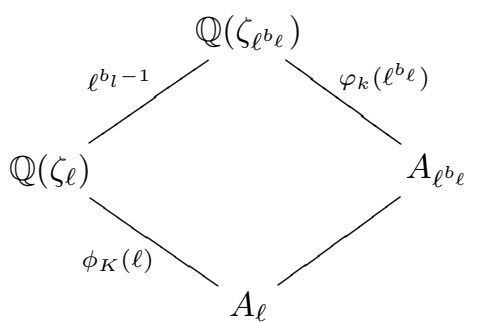

Observe that $A_{\ell}=K \cap \mathbb{Q}\left(\zeta_{\ell}\right)=A_{\ell^{b_{\ell}}} \cap \mathbb{Q}\left(\zeta_{\ell}\right)$. Since the compositum $A_{\ell^{b_{\ell}}} \mathbb{Q}\left(\zeta_{\ell}\right)$ is the smallest field containing both $A_{\ell^{b_{\ell}}}$ and $\mathbb{Q}\left(\zeta_{\ell}\right)$, we have that $\mathbb{Q}\left(\zeta_{\ell^{b_{\ell}}}\right) \supseteq A_{\ell^{b_{\ell}}} \mathbb{Q}\left(\zeta_{\ell}\right) \supseteq$ $\mathbb{Q}\left(\zeta_{\ell}\right)$. The Galois group $\operatorname{Gal}\left(\mathbb{Q}\left(\zeta_{\ell_{\ell}}\right) / \mathbb{Q}\left(\zeta_{\ell}\right)\right)$ is cyclic of order $\ell^{b_{\ell}-1}$. We then deduce that $A_{\ell^{b_{\ell}}} \mathbb{Q}\left(\zeta_{\ell}\right)=\mathbb{Q}\left(\zeta_{\ell^{j_{0}}}\right)$ for some $1 \leq j_{0} \leq b_{\ell}$. However, since $m_{K}$ is minimal, $b_{\ell}$ must be minimal as well. Therefore, we must have that $A_{\ell^{b} \ell} \nsubseteq \mathbb{Q}\left(\zeta_{\ell^{b_{\ell}-1}}\right)$. This implies that $A_{\ell^{b} \ell} \mathbb{Q}\left(\zeta_{\ell}\right)=\mathbb{Q}\left(\zeta_{\ell^{b} \ell}\right)$. Thus, from diagram (9), we see that $\varphi_{K}(\ell)=$ $\varphi_{K}\left(\ell^{b_{\ell}}\right)$. The claim in (8) follows since $\varphi_{K}\left(\ell^{j}\right)$ divides $\varphi_{K}\left(\ell^{j+1}\right)$ for all $j \geq 1$. The lemma follows by combining (7) with (8) .

The final goal of this section is to study the Dirichlet generating function

$$
D_{K}(s):=\sum_{n=1}^{\infty} \frac{1}{\varphi_{K}(n) n^{s-1}}
$$

and use it to prove two asymptotic identities involving the function $\varphi_{K}(n)$. Since $\varphi_{K}(n)$ agrees with $\varphi(n)$ for $\operatorname{gcd}\left(n, m_{K}\right)=1$, we begin with the Dirichlet series

$$
D(s):=\sum_{n=1}^{\infty} \frac{1}{\varphi(n) n^{s-1}}
$$

and introduce finitely many correction factors to obtain $D_{K}(s)$. Let $h(s)$ denote the Euler product

$$
h(s):=\prod_{\ell}\left\{1+\frac{1}{\ell^{s+2}}\left(1-\frac{1}{\ell^{s}}\right)\left(1-\frac{1}{\ell}\right)^{-1}\right\},
$$


and observe that, for any $\epsilon>0, h(s)$ is holomorphic and bounded for $\operatorname{Re}(s)>$ $-\frac{1}{2}+\epsilon$. Using the product formula for Euler's $\varphi$ function, we factor $D(s)$ as

$$
D(s)=\prod_{\ell}\left\{1+\frac{1}{\ell^{s}}\left(1-\frac{1}{\ell}\right)^{-1}\left(1-\frac{1}{\ell^{s}}\right)^{-1}\right\}=\zeta(s) \zeta(s+1) h(s),
$$

where again $\zeta(s)$ is the Riemann zeta function.

We now return to the Dirichlet series $D_{K}(s)$. In light of (10) and Lemma 1, for each prime $\ell$ dividing $m_{K}$, we define the correction factor

$$
D_{K, \ell}(s):=\frac{\left\{1+\frac{1}{\varphi_{K}(\ell) \ell^{s-1}}\left(1-\left(\frac{1}{\ell^{s-1}}\right)^{b_{\ell}-1}\right)\left(1-\frac{1}{\ell^{s-1}}\right)^{-1}+\frac{1}{\varphi_{K}(\ell)}\left(\frac{1}{\ell^{s-1}}\right)^{b_{\ell}}\left(1-\frac{1}{\ell^{s}}\right)^{-1}\right\}}{\left\{1+\frac{1}{\ell^{s}}\left(1-\frac{1}{\ell}\right)^{-1}\left(1-\frac{1}{\ell^{s}}\right)^{-1}\right\}},
$$

which has removable singularities at $s=0,1$ and is analytic elsewhere. We also define $D_{K, \ell}(0)$ (resp. $\left.D_{K, \ell}(1)\right)$ to be the limit of $D_{K, \ell}(s)$ as $s$ approaches 0 (resp. 1). In particular, we note that

$$
D_{K, \ell}(0)=\lim _{s \rightarrow 0} D_{K, \ell}(s)=\frac{\varphi\left(\ell^{b_{\ell}}\right)}{\varphi_{K}(\ell)}=\frac{\varphi\left(\ell^{b_{\ell}}\right)}{\varphi_{K}\left(\ell^{b_{\ell}}\right)} .
$$

Finally, from (10), we observe that $D_{K}(s)$ may be factored as

$$
D_{K}(s)=\zeta(s) \zeta(s+1) h(s) \prod_{\ell \mid m_{K}} D_{K, \ell}(s) .
$$

Lemma 2. For a fixed number field $K$, we have

$$
\begin{aligned}
\sum_{n<x}\left(1-\frac{n}{x}\right)^{2} \frac{1}{\varphi_{K}(n)} & =c_{1} \log x+c_{2}+\frac{\varphi\left(m_{K}\right)}{\varphi_{K}\left(m_{K}\right)} \frac{\log x}{x}+\frac{c_{3}}{x}+O\left(x^{-\frac{5}{4}}\right), \\
\sum_{n \leq x} \frac{1}{\varphi_{K}(n)} & =c_{1} \log x+c_{4}+O\left(\frac{1}{x}\right),
\end{aligned}
$$

where $c_{1}=\frac{\zeta(2) \zeta(3)}{\zeta(6)} \prod_{\ell \mid m_{K}} D_{K, \ell}(1)$, and $c_{2}, c_{3}, c_{4}$ are constants.

Proof. We begin with the proof of (13). For $c>0$,

$$
\begin{aligned}
\frac{1}{2} \sum_{n<x}\left(1-\frac{n}{x}\right)^{2} \frac{1}{\varphi_{K}(n)} & =\frac{1}{2 \pi i} \int_{c-i \infty}^{c+i \infty} D_{K}(s+1) \frac{x^{s}}{s(s+1)(s+2)} d s \\
& =R_{0}+R_{-1}+\frac{1}{2 \pi i} \int_{-\frac{5}{4}-i \infty}^{-\frac{5}{4}+i \infty} D_{K}(s+1) \frac{x^{s}}{s(s+1)(s+2)} d s,
\end{aligned}
$$

where $R_{0}$ and $R_{-1}$ are the residues of the integrand at $s=0$ and $s=-1$ respectively. See [11, Exercise 4.1.9, p. 57] for example. Using (12), we calculate the residues as follows:

$$
\begin{aligned}
R_{0} & =\frac{\zeta(2) h(1) \prod_{\ell \mid m_{K}} D_{K, \ell}(1)}{2} \log x+\frac{1}{2} c_{2}=\frac{c_{1}}{2} \log x+\frac{1}{2} c_{2}, \\
R_{-1} & =\frac{-\zeta(0) h(0) \prod_{\ell \mid m_{K}} D_{K, \ell}(0) \log x}{x}+\frac{c_{3}}{2 x}=\frac{\varphi\left(m_{K}\right)}{\varphi_{K}\left(m_{K}\right)} \frac{\log x}{2 x}+\frac{c_{3}}{2 x},
\end{aligned}
$$

where we have applied (11) to compute $\prod_{\ell \mid m_{K}} D_{K, \ell}(0)$ The remaining integral is clearly $O\left(x^{-5 / 4}\right)$. 
For the proof of (14), we begin with the formula

$$
\sum_{n \leq x} \frac{1}{\varphi_{K}(n)}=\frac{1}{2 \pi i} \int_{c-i \infty}^{c+i \infty} D_{K}(s+1) \frac{x^{s}}{s} d s
$$

and proceed in a manner similar to the proof of (13).

\section{Proof of Theorem 2}

Let $\theta_{K}(x):=\sum_{\mathrm{Np} \leq x} \log \mathrm{Np}$. We will frequently make use of the formula

$$
\theta_{K}(x)=x+O\left(x /(\log x)^{M}\right)
$$

throughout the remainder of this paper. The formula follows from (4). We now begin the proof of Theorem 2 by stating and proving the following lemma.

Lemma 3. For any $M>0$,

$$
\sum_{\mathbf{N} \mathfrak{p} \leq x} \sum_{N \mathfrak{p}^{\prime}=\mathrm{Np}}(\log \mathrm{N} \mathfrak{p})^{2}=[K: \mathbb{Q}](x \log x-x)+O\left(\frac{x}{(\log x)^{M}}\right) .
$$

Proof. First, note that since only finitely many rational primes may ramify in $K$, we only introduce an error of $O(1)$ by restricting our sum to prime ideals which do not lie above a rational prime ramifying in $K$. For a rational prime $p$, let $g_{p}$ denote the number of primes lying above $p$, let $f_{p}$ denote the degree of any prime lying above $p$, and let $e_{p}$ denote the ramification index of $p$ in $K$. Note that $e_{p}$ and $f_{p}$ are well-defined since $K / \mathbb{Q}$ is Galois. The contribution from the degree one primes gives us our main term. Thus, partial summation and (15) yield

$$
\begin{aligned}
\sum_{\mathbf{N} \mathfrak{p} \leq x} & \sum_{\substack{\mathfrak{p}^{\prime}=\mathrm{Np} \\
=}}(\log \mathrm{Np})^{2} \\
= & {[K: \mathbb{Q}] \sum_{\substack{p \leq x \\
e_{p}=1 \\
f_{p}=1}} g_{p}(\log p)^{2}+O(\sqrt{x} \log x) } \\
= & {[K: \mathbb{Q}] \log x\left(\theta_{K}(x)+O(\sqrt{x})\right)-[K: \mathbb{Q}] \int_{1}^{x} \frac{\theta_{K}(t)+O(\sqrt{t})}{t} d t } \\
= & {[K: \mathbb{Q}](x \log x-x)+O\left(x(\log x)^{-M}\right) . }
\end{aligned}
$$

Proof of Theorem 2. First, define

$$
S\left(x ; Q_{1}, Q_{2}\right):=\sum_{Q_{1}<q \leq Q_{2}} \sum_{a \in G_{q}}\left(\theta_{K}(x ; q, a)-\frac{x}{\varphi_{K}(q)}\right)^{2} .
$$

If $Q \leq x(\log x)^{-(M+1)}$, then Theorem 1 implies that $S(x ; 0, Q) \ll x^{2}(\log x)^{-M}$, and hence Theorem 2 follows since the error term dominates in this case. Thus, it suffices to consider the case when $Q>x(\log x)^{-(M+1)}$. Therefore, for the remainder of the proof, we will write $Q_{1}:=x(\log x)^{-(M+1)}$ and assume that $Q_{1}<Q_{2} \leq x$. By Theorem 1, we have

$$
S\left(x ; 0, Q_{2}\right)=S\left(x ; Q_{1}, Q_{2}\right)+O\left(x^{2}(\log x)^{-M}\right) .
$$


For $Q_{1}, Q_{2}$ as above,

$$
\begin{aligned}
& S\left(x ; Q_{1}, Q_{2}\right) \\
& =\sum_{Q_{1}<q \leq Q_{2}} \sum_{a \in G_{q}}\left\{\theta_{K}(x ; q, a)^{2}-\frac{2 x}{\varphi_{K}(q)} \theta_{K}(x ; q, a)+\frac{x^{2}}{\varphi_{K}(q)^{2}}\right\} \\
& =\sum_{Q_{1}<q \leq Q_{2}}\left\{\sum_{a \in G_{q}} \theta_{K}(x ; q, a)^{2}-\frac{x}{\varphi_{K}(q)}\left(\begin{array}{c}
\left.\left.2 \theta_{K}(x)-2 \sum_{\substack{\mathrm{Np} \leq x,(\mathrm{~Np}, q)>1}} \log \mathrm{Np}-x\right)\right\} \\
=\sum_{Q_{1}<q \leq Q_{2}} \sum_{a \in G_{q}} \theta_{K}(x ; q, a)^{2}-x^{2} \sum_{Q_{1}<q \leq Q_{2}} \frac{1}{\varphi_{K}(q)}+O\left(\frac{x^{2}}{(\log x)^{M}}\right) .
\end{array}\right.\right.
\end{aligned}
$$

Now, observe that

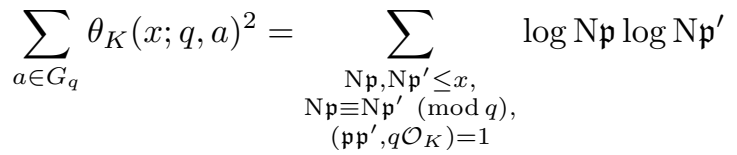

$$
\begin{aligned}
& =\sum_{\substack{\mathrm{N} \mathfrak{p}=\mathrm{Np}^{\prime} \leq x,\left(\mathfrak{p} \mathfrak{p}^{\prime}, q \mathcal{O}_{K}\right)=1}}(\log \mathrm{Np})^{2}+\sum_{\substack{\mathrm{Np}, \mathrm{Np}^{\prime} \leq x ; N \mathfrak{N} \neq \mathrm{Np}^{\prime}, N \mathfrak{p} \equiv \mathbb{N p}^{\prime}(\bmod q)}} \log \mathrm{Np} \log \mathrm{Np}^{\prime} .
\end{aligned}
$$

Note that removing the condition $\left(\mathfrak{p p}^{\prime}, q \mathcal{O}_{K}\right)=1$ from the second sum is justified. For example, if $\mathfrak{p} \mid q \mathcal{O}_{K}$ and $p$ lies below $\mathfrak{p}$, then the condition $\mathrm{Np} \equiv \mathrm{Np}^{\prime}(\bmod q)$ implies that $0 \equiv \mathrm{Np}^{\prime}(\bmod ) p$. This in turn implies that $\mathrm{Np}=\mathrm{Np}^{\prime}$. Thus, we define

$$
\begin{aligned}
& H\left(x ; Q_{1}, Q_{2}\right):=\sum_{Q_{1}<q \leq Q_{2}} \sum_{\substack{\mathrm{N} \mathfrak{p}=\mathrm{Np}^{\prime} \leq x,\left(\mathfrak{p}^{\prime}, q \mathcal{O}_{K}\right)=1}}(\log \mathrm{Np})^{2} ; \\
& J\left(x ; Q_{1}, Q_{2}\right):=\sum_{Q_{1}<q \leq Q_{2}} \sum_{\substack{\mathrm{Np}, N \mathfrak{p}^{\prime} \leq x ; \mathrm{Np} \neq \mathrm{Np}^{\prime}, \mathrm{Np} \equiv \mathrm{Np}^{\prime}(\bmod q)}} \log \mathrm{Np} \log \mathrm{Np}^{\prime} .
\end{aligned}
$$

Now (17) may be rewritten as

$$
\begin{aligned}
S\left(x ; Q_{1}, Q_{2}\right)=H\left(x ; Q_{1}, Q_{2}\right) & +J\left(x ; Q_{1}, Q_{2}\right) \\
& -c_{1} x^{2} \log \left(Q_{2} / Q_{1}\right)+O\left(\frac{x^{2}}{(\log x)^{M}}\right) .
\end{aligned}
$$

Note that we have applied the second part of Lemma 2 to the second term of (17).

Removing the condition $\left(\mathfrak{p p}^{\prime}, q \mathcal{O}_{K}\right)=1$ from the inner sum of $H\left(x ; Q_{1}, Q_{2}\right)$ introduces an error which is $O\left((\log x)^{2}\right)$. Thus, we may apply Lemma 3 to obtain

$$
\begin{aligned}
H\left(x ; Q_{1}, Q_{2}\right) & =\left\{Q_{2}-Q_{1}+O(1)\right\}\left\{[K: \mathbb{Q}](x \log x-x)+O\left(x(\log x)^{-M}\right)\right\} \\
& =[K: \mathbb{Q}] x Q_{2} \log x-[K: \mathbb{Q}] x Q_{2}+O\left(\frac{x^{2}}{(\log x)^{M}}\right) .
\end{aligned}
$$


Now, define $J(x ; Q):=J(x ; Q, x)$, so that $J\left(x ; Q_{1}, Q_{2}\right)=J\left(x ; Q_{1}\right)-J\left(x ; Q_{2}\right)$. Then

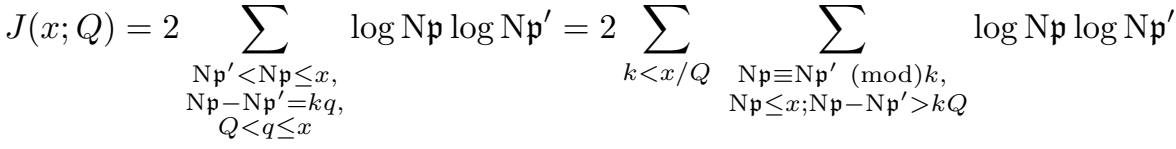

$$
\begin{aligned}
& =2 \sum_{k<x / Q} \sum_{a \in G_{k}} \sum_{\begin{array}{c}
N \mathfrak{p}^{\prime}<x-k Q, \\
\mathrm{~Np}^{\prime} \equiv a(\bmod k)
\end{array}} \log \mathrm{Np}^{\prime} \sum_{\begin{array}{c}
k Q+N \mathfrak{p}^{\prime}<\mathrm{Np} \leq x, \\
\mathrm{~Np} \equiv a(\bmod k)
\end{array}} \log \mathrm{Np} .
\end{aligned}
$$

Since $Q \geq Q_{1}=x /(\log x)^{M+1}$, we have $k<x / Q \leq(\log x)^{M+1}$ and $k Q \geq$ $x /(\log x)^{M+1}$. Thus, we may apply (4) and write

$$
\theta_{K}(x ; a, k)-\theta_{K}\left(k Q+\mathrm{Np}^{\prime} ; a, k\right)=\frac{x-k Q-\mathrm{Np}^{\prime}}{\varphi_{K}(k)}+O\left(\frac{x}{(\log x)^{2 M+1}}\right)
$$

for the innermost sum above. This gives

$$
\begin{aligned}
J(x, Q)= & 2 \sum_{k<\frac{x}{Q}} \frac{1}{\varphi_{K}(k)} \sum_{\begin{array}{c}
\mathrm{Np}^{\prime}<x-k Q, \\
\left(\mathrm{~Np}^{\prime}, k\right)=1
\end{array}}\left(x-k Q-\mathrm{Np}^{\prime}\right) \log \mathrm{Np}^{\prime} \\
& +O\left(\frac{x}{(\log x)^{2 M+1}} \sum_{k<\frac{x}{Q}} \theta_{K}(x)\right) \\
= & 2 \sum_{k<\frac{x}{Q}} \frac{\int_{1}^{x-k Q} \theta_{K}(t) d t}{\varphi_{K}(k)}+O\left(x \sum_{k<\frac{x}{Q}} \frac{\log k}{\varphi_{K}(k)}\right)+O\left(\frac{x^{3}}{Q(\log x)^{2 M+1}}\right),
\end{aligned}
$$

where the last line follows by partial summation applied to the inner sum of the main term. Therefore, by (15), we have

$$
J(x, Q)=x^{2} \sum_{k<\frac{x}{Q}}\left(1-\frac{k Q}{x}\right)^{2} \frac{1}{\varphi_{K}(k)}+O\left(\frac{x^{2}}{(\log x)^{M}}\right) .
$$

We consider two different cases for the treatment of $J\left(x ; Q_{1}, Q_{2}\right)$. First, if $Q_{2}=$ $x$, then

$$
\begin{aligned}
J\left(x ; Q_{1}, Q_{2}\right) & =J\left(x ; Q_{1}\right) \\
& =x^{2}\left\{c_{1} \log \left(x / Q_{1}\right)+c_{2}+O\left(\frac{\log \left(x / Q_{1}\right)}{x / Q_{1}}\right)\right\}+O\left(\frac{x^{2}}{(\log x)^{M}}\right) \\
& =c_{1} x^{2} \log \left(Q_{2} / Q_{1}\right)+c_{2} x^{2}+O\left(\frac{x^{2}}{(\log x)^{M}}\right) .
\end{aligned}
$$

In the case that $Q_{2} \leq x$ (including the previous case), we may write

$$
\begin{aligned}
J\left(x ; Q_{1}, Q_{2}\right)= & J\left(x ; Q_{1}\right)-J\left(x ; Q_{2}\right) \\
= & c_{1} x^{2} \log \left(Q_{2} / Q_{1}\right)-\frac{\varphi\left(m_{K}\right)}{\varphi_{K}\left(m_{K}\right)} x Q_{2} \log \left(x / Q_{2}\right)-c_{3} x Q_{2} \\
& +O\left(x^{3 / 4} Q_{2}^{5 / 4}\right)+O\left(\frac{x^{2}}{(\log x)^{M}}\right) .
\end{aligned}
$$

Theorem 2 now follows by combining (16), (18), (19), (20), and (21). 


\section{ACKNOWLEDGMENT}

The author is grateful to Andrew Granville both for suggesting this work and for pointing him toward the paper of Hooley [7, which was so very helpful.

\section{REFERENCES}

[1] M.B. Barban. On the distribution of primes in arithmetic progressions "on average". Dokl. Akad. Nauk SSSR, 5:5-7, 1964 (Russian).

[2] H. Davenport and H. Halberstam. Primes in arithmetic progressions. Michigan Math. J., 13:485-489, 1966. MR0200257 (34:156)

[3] H. Davenport and H. Halberstam. Corrigendum: "Primes in arithmetic progression". Michigan Math. J., 15:505, 1968. MR0233778 (38:2099)

[4] Harold Davenport. Multiplicative Number Theory. Springer-Verlag, New York, 1980. MR606931 (82m:10001)

[5] Larry Joel Goldstein. A generalization of the Siegel-Walfisz theorem. Trans. Amer. Math. Soc., 149:417-429, 1970. MR0274416(43:181)

[6] Jürgen G. Hinz. On the theorem of Barban and Davenport-Halberstam in algebraic number fields. J. Number Theory, 13(4):463-484, 1981. MR642922 (83g:10032)

[7] Christopher Hooley. On the Barban-Davenport-Halberstam theorem. I. J. Reine Angew. Math., 274/275:206-223, 1975. Collection of articles dedicated to Helmut Hasse on his seventyfifth birthday, III. MR0382202 (52:3090a)

[8] Serge Lang. Algebraic number theory, volume 110 of Graduate Texts in Mathematics. Springer-Verlag, New York, second edition, 1994. MR1282723 (95f:11085)

[9] A. F. Lavrik. On the twin prime hypothesis of the theory of primes by the method of I. M. Vinogradov. Soviet Math. Dokl., 1:700-702, 1960. MR0157955 (28:1183)

[10] H. L. Montgomery. Primes in arithmetic progressions. Michigan Math. J., 17:33-39, 1970. MR0257005 (41:1660)

[11] M. Ram Murty. Problems in analytic number theory, volume 206 of Graduate Texts in Mathematics. Readings in Mathematics. Springer-Verlag, New York, 2001. MR:1803093 (2001k:11002)

[12] Ethan Smith. A generalization of the Barban-Davenport-Halberstam Theorem to number fields. J. Number Theory, 129(11):2735-2742, 2009. MR.2549528

[13] Robin J. Wilson. The large sieve in algebraic number fields. Mathematika, 16:189-204, 1969. MR0263774(41:8374)

Department of Mathematical Sciences, Michigan Technological University, 1400 Townsend Drive, Houghton, Michigan 49931-1295

E-mail address: ethans@mtu.edu

$U R L:$ www.math.mtu.edu/ ${ }^{\sim}$ ethans 